\title{
Wormhole geometry from real feasible matter sources
}

\author{
F.Rahaman*, M.Kalam ${ }^{\dagger}$ and K A Rahman*
}

\begin{abstract}
We provide a prescription of real feasible sources that supply fuel to construct a traversable wormhole. A class of exact solutions for Einstein-Maxwell field equations describing wormhole with an anisotropic matter distribution has been presented. The anisotropy plays a crucial role for the existence of the spacetime comprising wormhole geometry.
\end{abstract}

\section{Introduction:}

During last 30 years, more than 700 articles [1] have been written on wormholes after the pioneering work of Morris and Thorne [2]. Scientists show interest on wormhole physics because it opens up a possibility to construct a time machine [3]. It is well known that wormhole demands exotic matter ( matter that violates weak or null energy conditions [WEC or NEC] ). There are several proposals have been proposed regarding the exotic matter sources that support wormhole spacetime. But scientists remain silent whether it is possible to manufacture or creation of this exotic matter. As exotic matter violates the NEC, so it is quite impossible to provide such type of matter source to construct a wormhole. In other words, time machine ( which is a possible consequence of wormhole ) can never be constructed. In this letter we will give a clue how one can get feasible matter sources that supply fuel to construct and sustain a wormhole. In the present investigation, we will show that if we are supplied anisotropic matter source and electromagnetic field, then one could construct a traversable wormhole.

\footnotetext{
0 *Dept.of Mathematics, Jadavpur University, Kolkata-700 032, India

E-Mail:farook_rahaman@yahoo.com

$\nmid$ Dept. of Phys. , Netaji Nagar College for Women, Regent Estate, Kolkata-700092, India.

E-Mail:mehedikalam@yahoo.co.in
} 
Anisotropic matter sources are not absurd ( rather we say, not exotic ). Recent observations on highly compact astrophysical objects like X ray pulsar Her X-1, X ray buster $4 \mathrm{U}$ 1820-30, millisecond pulsar SAX J 1808.4 - 3658, X ray sources 4U 1728 - 34 etc indicate that the densities of such objects are normally beyond nuclear matter density. Theoretical advances in the last decades indicate that pressures within such stars are anisotropic i.e. radial pressure $\left(p_{r}\right)$ is not equal to tangential pressure $\left(p_{t}\right)$ in such bodies [4].

If an advanced engineer imbued with new ideas will able to produce anisotropic matter source ( like above astrophysical compact objects ), then wormhole could be constructed physically. Rather producing negative energy or matter that violates NEC, we think, it is simpler to creation compact object with density greater than the nuclear matter density.

\section{Basic equations for constructing wormholes:}

For the present study the metric for static spherically symmetric spacetime is taken as

$$
d s^{2}=-e^{\nu(r)} d t^{2}+e^{\mu(r)} d r^{2}+r^{2}\left(d \theta^{2}+\sin ^{2} \theta d \phi^{2}\right)
$$

The most general energy momentum tensor compatible with spherically symmetry is

$$
T_{\nu}^{\mu}=\left(\rho+p_{t}\right) u^{\mu} u_{\nu}-p_{t} g_{\nu}^{\mu}+\left(p_{r}-p_{t}\right) \eta^{\mu} \eta_{\nu}
$$

with

$u^{\mu} u_{\mu}=-\eta^{\mu} \eta_{\mu}=1$

The Einstein-Maxwell field equations for the above spherically symmetric metric corresponding to the charged anisotropic matter distribution are given by

$$
\begin{gathered}
e^{-\mu}\left[\frac{\mu^{\prime}}{r}-\frac{1}{r^{2}}\right]+\frac{1}{r^{2}}=8 \pi \rho+E^{2} \\
e^{-\mu}\left[\frac{1}{r^{2}}+\frac{\nu^{\prime}}{r}\right]-\frac{1}{r^{2}}=8 \pi p_{r}-E^{2} \\
e^{-\mu}\left[\frac{1}{2}\left(\nu^{\prime}\right)^{2}+\nu^{\prime \prime}-\frac{1}{2} \mu^{\prime} \nu^{\prime}+\frac{1}{r}\left(\nu^{\prime}-\mu^{\prime}\right)\right]=8 \pi p_{t}+E^{2}
\end{gathered}
$$

and

$$
\left(r^{2} E\right)^{\prime}=4 \pi r^{2} \sigma e^{\frac{\mu}{2}}
$$

Equation (6) can equivalently be expressed in the form

$$
E(r)=\frac{1}{r^{2}} \int_{0}^{r} 4 \pi r^{2} \sigma e^{\frac{\mu}{2}} d r=\frac{q(r)}{r^{2}}
$$

where $q(r)$ is the total charge of the sphere under consideration. 
Also, the conservation equation is given by

$$
\frac{d p_{r}}{d r}+\left(\rho+p_{r}\right) \frac{\nu^{\prime}}{2}=\frac{1}{8 \pi r^{4}} \frac{d q^{2}}{d r}+\frac{2\left(p_{t}-p_{r}\right)}{r}
$$

Here, $\rho, E, \sigma$ and $q$ are respectively the matter energy density, electric field strength, electric charged density and electric charge and $\Delta=p_{t}-p_{r}$, is the measure of anisotropy. The prime denotes derivative with respect to ' $r$ '.

Solutions: To control the solutions, we assume the following assumptions:

(a)

$$
\nu(r)=0
$$

Argument: One of the traversability properties is that the tidal gravitational forces experienced by a traveller must be reasonably small. So, we assume a zero tidal force as seen by the stationary observer. Thus one of the traversability conditions is automatically satisfied.

$$
\Delta=p_{t}-p_{r}=-\alpha q^{2} r^{n}
$$

where, $\alpha>0$ and $\mathrm{n}$ are arbitrary constants.

Argument: According to Usov [5], the stars whose density beyond the nuclear matter density, the anisotropy within such stars could be the presence of strong electromagnetic field. So, the above assumption is justified.

(c)

$$
\sigma e^{\frac{\mu}{2}}=\sigma_{0} r^{s}
$$

$\left(\sigma_{0}\right.$ and $\mathrm{s}$ are arbitrary constants )

Argument: In usual sense, the term $\sigma e^{\frac{\mu}{2}}$ occurring inside the integral sign in the equation (7), is called the volume charge density and hence the condition $\sigma e^{\frac{\mu}{2}}=\sigma_{0} r^{s}$, can equivalently be interpreted as the volume charge density being polynomial function of ' $r$ '. The constant $\sigma_{0}$ is the charge density at $r=0$, the center of the charged matter [6].

$$
p_{r}=m \rho
$$

Argument: The above equation indicates the equation of state with $0<m<1$. 
Taking into account of equations (9) - (12), one gets the following solutions of the field equations (3) - (8) as

$$
\begin{aligned}
& q^{2}(r)=\frac{16 \pi^{2} \sigma_{0}^{2}}{(s+3)^{2}} r^{2 s+6} \\
& E^{2}(r)=\frac{16 \pi^{2} \sigma_{0}^{2}}{(s+3)^{2}} r^{2 s+2} \\
& \rho=A r^{2 s+2}+B r^{2 s+n+6}
\end{aligned}
$$

where $A=\frac{4 \pi \sigma_{0}^{2}}{m(s+3)(2 s+2)}$ and $B=\frac{-32 \pi^{2} \alpha \sigma_{0}^{2}}{m(s+3)^{2}(2 s+n+6)}$.

$$
e^{-\mu}=1-\frac{b(r)}{r}
$$

where,

$$
b(r)=F r^{u+1}+X r^{w+1}
$$

where, $w=2 s+4, u=2 s+n+8, X=\frac{32 \pi^{2} \sigma_{0}^{2}}{m(s+3)(2 s+2)(2 s+5)}+\frac{16 \pi^{2} \sigma_{0}^{2}}{(s+3)^{2}(2 s+5)}$ and $F=\frac{-256 \pi^{3} \alpha \sigma_{0}^{2}}{m(s+3)^{2}(2 s+n+6)(2 s+n+9)}$

\section{Wormhole structure:}

For the assumption $\nu(r)=0$ implies no horizon exists in the spacetime. Since the space time is asymptotically flat i.e. $\frac{b(r)}{r} \rightarrow 0$ as $|r| \rightarrow \infty$, the Eq.(17) is consistent only when $2 s+n+8<0$ and $2 s+4<0$.

Also, one can note that $\mathrm{X}$ is negative for the above restrictions and as $|r| \rightarrow \infty, q^{2}(r)$ and $E^{2}(r) \rightarrow 0$, so one has to take the following restrictions on 's' to get wormhole structure as $s<-3,2 s+n+8<0$ but $2 s+n+9>0$.

Here the throat occurs at $r=r_{0}$ for which $b\left(r_{0}\right)=r_{0}$ i.e. $1=F r_{0}^{u}+X r_{0}^{w}$. For the suitable choices of the parameters, the graph of the function $G(r)=b(r)-r$ indicates the point $r_{0}$ , where G(r) cuts the 'r' axis (see Fig. 1 ). From the graph, one can also note that when $r>r_{0}, G(r)<0$ i.e. $b(r)-r<0$. This implies $\frac{b(r)}{r}<1$ when $r>r_{0}$. Also, from the graph, we see that $\mathrm{G}$ is a decreasing function of $\mathrm{r}$ and hence $G^{\prime}(r)<0$. In other words, $b^{\prime}\left(r_{0}\right)<1$ i.e. flare-out condition has been satisfied. 


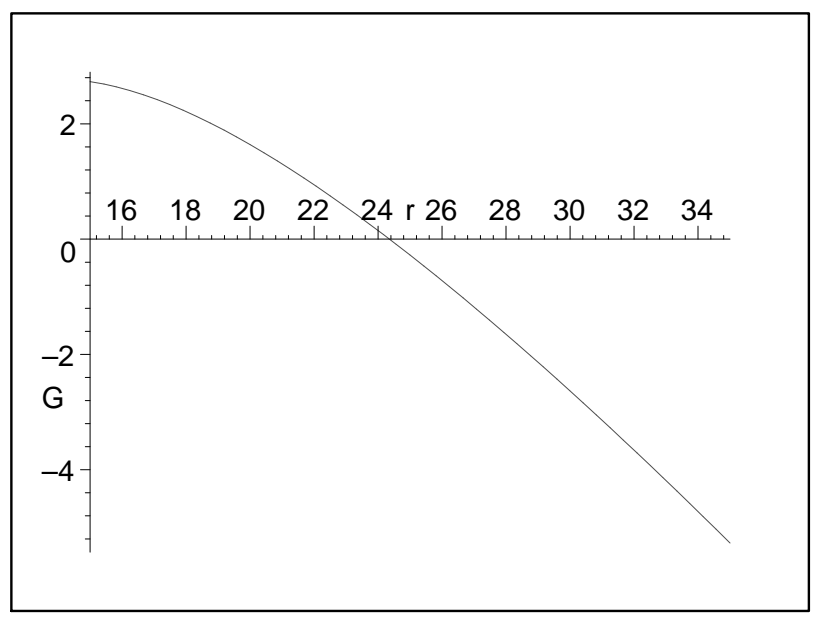

Figure 1: Throat occurs where $G(r)$ cuts 'r' axis ( choosing suitably the parameters as $\left.\sigma_{0}=1, \alpha=.0001, s=-3.5, n=-1.5, m=.5\right)$.

Thus our solution describing a static spherically symmetric wormhole supported by anisotropic matter distribution in presence of electromagnetic field. The axially symmetric embedded surface $z=z(r)$ shaping the Wormhole's spatial geometry is a solution of

$$
\frac{d z}{d r}= \pm \frac{1}{\sqrt{\frac{r}{b(r)}-1}}
$$

One can note from the definition of Wormhole that at $r=r_{0}$ (the wormhole throat) Eq.(18) is divergent i.e. embedded surface is vertical there. One can see that embedding diagram of this wormhole ( expand binomially in powers of $r$ and retaining a few terms) in Fig-2. The surface of revolution of the curve about the vertical $\mathrm{z}$ axis makes the diagram complete (see Fig.3).

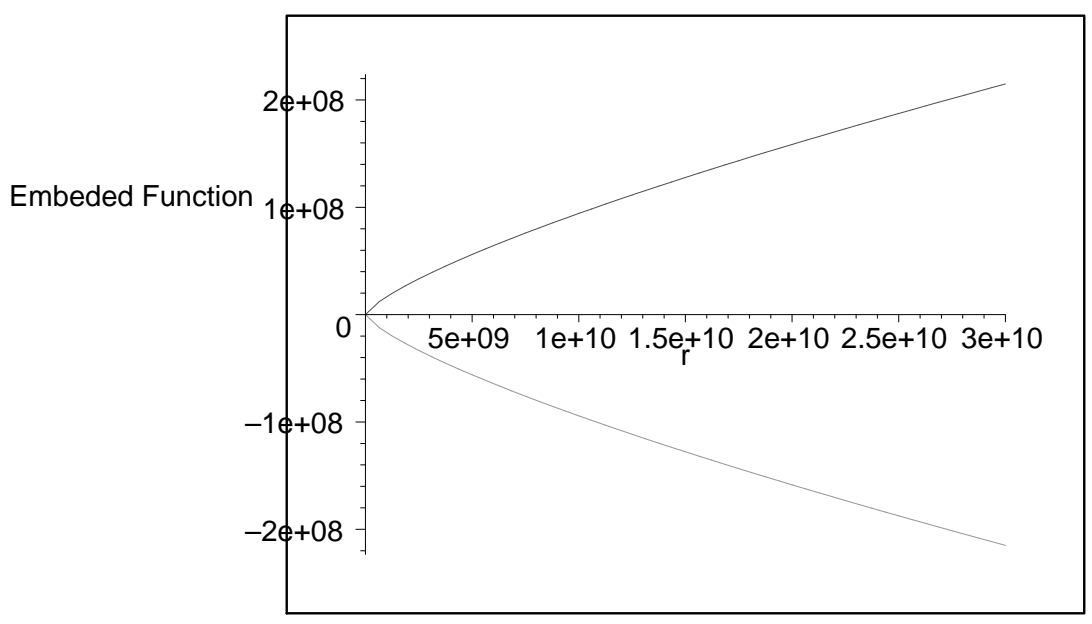

Figure 2: The embedding diagram of the wormhole( retaining a few terms and choosing suitably the parameters as $\sigma_{0}=1, \alpha=.0001, s=-3.5, n=-1.5, m=.5$ ). 


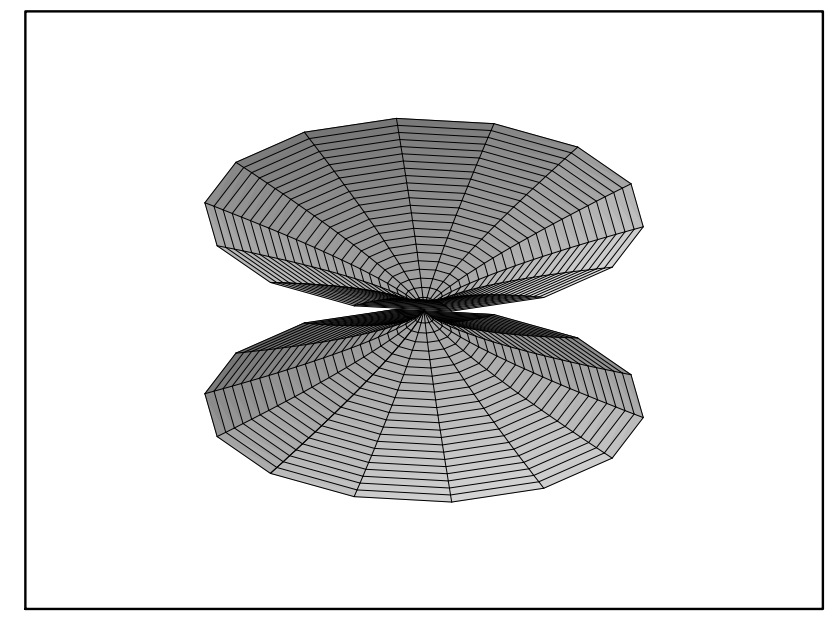

Figure 3: The full visualization of the surface generated by the rotation of the embedded curve ( retaining a few terms ) about the vertical $\mathrm{z}$ axis.

\section{Final Remarks:}

In this article, we provide matter sources that give birth to wormhole like geometry. By considering the anisotropy of the matter distribution, we have shown how wormhole could be constructed in presence of electromagnetic field. We have presented an example of the possible structure of the wormhole generated by the rotation of the embedded curve about the vertical $\mathrm{z}$ axis. One can see that $\rho_{\text {effective }}>0, \rho_{\text {effective }}+p_{\text {r effective }}>0, \rho_{\text {effective }}+$ $p_{t \text { effective }}>0$ for all $r>r_{0}$ i.e. all energy conditions are satisfied out side the wormhole throat. But at the throat i.e. at $r=r_{0}$, NEC is violated. Nevertheless, this wormhole has been constructed by real feasible matter sources. Although several assumptions were considered, we give valid arguments against it. We hope scientists would be motivated by our approach and in future, will try to find sophisticated way for constructing feasible wormhole. 


\section{Acknowledgments}

F.R is thankful to Jadavpur University and DST , Government of India for providing financial support. MK has been partially supported by UGC, Government of India under MRP scheme.

\section{References}

[1] See the website: www.slac.stanford.edu/spires/hep

[2] M. Morris and K. Thorne , American J. Phys. 56, 39 (1988)

[3] M. Visser, Lorentzian Wormholes: From Einstien to Hawking, AIP Press (1995)

[4] Sharma. R and Maharaj. S, gr-qc/ 0702046 ( Mon.Not.Roy.Astron.Soc.375:12651268,2007 and references therein. )

[5] Usov.V , Phys.Rev.D 70, 067301 (2004)

[6] Bronnikov K A et al, Class.Quan.Grav. 20, 3797 (2003); Tiwari R et al, Ind.J.Pure and Appl.Maths., 27, 907 (1996); Wang P et al,Class.Quan.Grav. 20, 3797 (2005); Sola J et al, Mod.Phys.Lett.A, 21, 479(2006) 